\title{
Strates
}

STRATES Matériaux pour la recherche en sciences sociales

5 | 1990

Conjuguer stratégies et territoires?

\section{Protection de la nature et gestion agricole dans le Parc national des Cévennes}

\section{Sylvie Mousset}

\section{(2) OpenEdition \\ 1 Journals}

\section{Édition électronique}

URL : http://journals.openedition.org/strates/1490

DOI : $10.4000 /$ strates. 1490

ISSN : $1777-5442$

Éditeur

Laboratoire Ladyss

Édition imprimée

Date de publication : 31 décembre 1990

ISSN : 0768-8067

\section{Référence électronique}

Sylvie Mousset, "Protection de la nature et gestion agricole dans le Parc national des Cévennes », Strates [En ligne], 5 | 1990, mis en ligne le 16 mars 2007, consulté le 08 septembre 2020. URL : http:// journals.openedition.org/strates/1490; DOI : https://doi.org/10.4000/strates.1490

Ce document a été généré automatiquement le 8 septembre 2020.

Tous droits réservés 


\title{
Protection de la nature et gestion agricole dans le Parc national des Cévennes ${ }^{1}$
}

\author{
Sylvie Mousset
}

1 Depuis ces dernières années, le binôme agriculture/environnement est placé au rang des actualités brûlantes - tant sur la scène politique que sur la scène scientifique - à l'échelle de l'Europe tout entière. En témoignent:

2 - l'impact et l'audience recueillies auprès des instances chargées de l'environnement et du développement agricole par le Colloque européen organisé en 1986 par la Fédération française des sociétés de protection de la nature (F.F.S.P.N.). Responsables de l'agriculture et acteurs de l'environnement ont, ensemble, examiné les solutions capables d'éviter l'abandon rural et confronté les expériences concernant l'intégration des concepts de l'Écologie dans les stratégies locales de développement agricole et plus largement rural. Chacun s'est mobilisé sur la question des nouvelles fonctions qui doivent être reconnues aux agriculteurs des régions déprimées et sur leur comptabilité avec une politique de protection du patrimoine naturel.

3 - la prise de conscience de plus en plus aigue - transparaissant dans les discours du ministère de l'Environnement - qu'il ne peut plus être pratiqué de politique de protection de la nature sans tenir compte de la politique agricole et de son évolution. Inversement, la publication par la Communauté européenne en 1985 du Livre Vert dans lequel est affirmée "la volonté de soutenir l'activité agricole dans les zones où elle est indispensable pour la sauvegarde de l'environnement", montre que les préoccupations d'ordre naturel pénètrent les instances agricoles nationales et européennes. Défense de la nature, défense de l'agriculture, même combat?

4 La question du rapport agriculture/environnement est complexe. Elle a déjà engendré nombre d'analyses et suscité de multiples débats ${ }^{2}$, à l'image de la multiplicité des problématiques posées. Tantôt il s'agit de la qualité des sols, tantôt de la qualité des paysages, du milieu de vie (pollution des nappes) ou encore de la qualité des produits agricoles. C'est une question qui revêt d'innombrables aspects selon le type 
d'agriculture et selon la spécificité de l'espace où elle est appréhendée. Enfin, elle est l'objet de discours et de pratiques contradictoires.

Quelle que soit sa complexité, c'est une question privilégiée pour le géographe: ne permet-elle pas, en effet, de recentrer notre réflexion sur la place du milieu naturel dans la gestion d'un territoire par une société donnée - thème majeur de la géographie - sachant que les formes des rapports entre les cadres naturels de la vie sociale et le comportement des groupes humains varient à l'infini dans l'espace et dans le temps?

6 Après un premier travail portant sur l'un des contentieux classiques agriculture/ environnement, lié à la question de l'intensification culturale en zone agricole à haute productivité, l'ambition initiale de notre recherche était une approche du rapport agriculture/environnement dans des zones fragiles d'un point de vue naturel, marginalisées d'un point de vue économique et donc particulièrement sensibles aux évolutions économiques et politiques. Notre étude se voulait être, face aux préoccupations actuelles, l'esquisse d'une réponse à une demande sociale, à un besoin d'action et d'expérimentation.

7 Avec pour cadre d'étude le Parc national des Cévennes, ce parc dit exceptionnel, car habité et, de surcroît, exploité en sa zone centrale - zone soumise à une réglementation stricte de protection - par des agriculteurs encore en activité, nous disposions d'un espace privilégié pour analyser, dans le champ élargi énoncé ci-dessus, un aspect jusqu'ici peu abordé : la liaison qui peut exister entre la gestion d'un territoire et les problèmes de protection de la nature. Notre question centrale était : quelle peut-être et quelle doit être la place de l'agriculture comme composante de la gestion d'un territoire par rapport à la question de la protection de la nature ?

8 Tout un ensemble de thèmes de réflexion en découle: politique de protection et politique territoriale, gestion agricole et gestion de la nature - qu'en est-il de cette vieille antinomie entre agriculture et protection par laquelle les forestiers justifièrent leurs activités dès la fin du XIXe siècle ? en quels termes ce rapport est-il aujourd'hui appréhendé dans le cadre d'un Parc national?-, promotion de l'agriculture et promotion des richesses naturelles et culturelles: est-ce une gestion du possible et quelles en sont les limites?

9 Ces interrogations mettent au centre de notre analyse les notions de marginalité et d'intégration qui, appliquées à un espace agricole, introduisent une relation à l'environnement tout à fait différente.

10 Dès le départ, des jalons nous étaient posés : notre travail s'intègre, en effet, dans un Programme interdisciplinaire de recherches sur l'environnement mis en place en 1981 sur 24 communes cévenoles. Outre que celui-ci nous offrait le cadre scientifique pour une analyse des rapports de l'homme à la nature en milieu rural, il nous confortait dans la pertinence d'une nécessaire compréhension des problèmes de développement et d'aménagement en termes d'environnement.

11 L'ambition théorique de ce programme est d'appréhender les interactions existant entre écosystèmes et systèmes sociaux et de concevoir un "système permanent d'observation des changements écologiques, économiques et sociaux afin de définir les règles d'une gestion dite "gestion écologique" qui tienne davantage compte des contraintes, des potentialités et des dynamiques écologiques et sociales des milieux concernés ». 
12 Il s'agissait de mener une étude approfondie des interactions entre tous les systèmes en présence (dynamiques écologiques, économiques et sociales). Tandis que toute une équipe pluridisciplinaire était chargée de passer au décryptage de l'analyse chacun de ces systèmes, une inconnue demeurait: le Parc national des Cévennes en tant que composante d'une gestion dite " écologique » du territoire.

Il nous revenait alors de déterminer le rôle institutionnel du Parc en tant qu'acteur intervenant dans l'évolution des hautes terres cévenoles et d'évaluer les changements écologiques et sociaux en interrelation avec l'intervention de l'État dans un domaine particulier, celui de la protection de la nature.

14 La particularité de cette institution, et surtout de l'espace à protéger et à gérer, est apparue telle que l'analyse des politiques et stratégies d'État pour préserver la nature et la culture cévenoles induisait nécessairement et simultanément une analyse du rapport agriculture/environnement.

S'interroger sur la mise en place d'une "politique agricole » dans un Parc national et sur sa signification - enjeu de développement? enjeu paysager? - supposait aussi de mieux cerner les conditions économiques et sociales dans lesquelles le Parc " protecteur de la nature » est entré sur la scène locale, venait bouleverser le système de pouvoir départemental et régional largement dominé par les organisations agricoles principalement lozériennes.

16 Le Parc apparaissant toujours sur "le territoire des autres " ${ }^{3}$, comment s'articulent les divers pouvoirs en présence, chacun secrétant ses propres projets fondés sur sa propre représentation de la nature et du développement agricole ? À titre d'exemple, l'action de la Direction départementale de l'Agriculture (D.D.A.) s'inscrit dans le cadre d'une doctrine officielle nationale qui contient un projet de développement agricole propre : l'agriculture des Cévennes est globalement vue comme une agriculture de montagne, qui souffre de certains handicaps par rapport à l'agriculture de plaine; le schéma correspond aux observations que l'on peut faire sur la réalité cévenole, celle-ci étant en fait le résultat de la mise en place de ce schéma.

17 Face à cette représentation, il y a celle du parc dont la mission de protection nécessite la présence d'un tissu agricole dynamique et valorisé dans son travail : quels vont être les moyens d'intervention, institutionnels et financiers, des responsables du Parc? De cette confrontation, quelles conséquences induites par la valorisation des ressources cévenoles?

18 Au terme de cette recherche sur une région, rappelons-le, parmi les plus étudiées de France, trois principales conclusions doivent être soulignées :

19 1. L'histoire du Parc national des Cévennes montre, plus que tout autre, combien le phénomène "parc » est en grande partie un phénomène rural. La plupart des études sur les Parcs nationaux se complaisent à mettre en valeur l'origine urbaine de ces structures. Or, la part rurale est tout autant, sinon plus, importante dans leur genèse. Ils sont, en effet, le produit de communautés rurales en pleine mutation, communautés agropastorales dont l'espace à usages multiples est hétérogène.

Une rétrospective historique de la protection de la nature en France permet d'établir un rapprochement intéressant entre la Loi d'Orientation agricole et la Loi sur la Création des Parcs nationaux, toutes deux de la même année et discutée à l'Assemblée nationale lors des mêmes débats. 
21 La création des Parcs est le résultat de la "révolution silencieuse », de l'apparition d'un nouveau type d'agriculteur - 1960, ne sommes-nous pas au cœur de la restructuration agricole?

Ces propos prennent d'autant plus d'ampleur en Cévennes que le Parc national a été mis en place en 1970 sur un espace, rappelons-le, habité et constitué d'unités agricoles considérées par l'État comme non viables et vouées à terme à disparaître, ce qui les prédisposait à une première expérimentation du Plan Vedel (1969): un plan qui associait les agriculteurs des zones dites défavorisées à des « jardiniers de la nature » et leur préconisait des sources complémentaires de revenu grâce aux activités de détente et de loisirs qui s'y seraient développées. Ils auraient ainsi pu consacrer leur temps à la protection et à l'exploitation du domaine rural indépendamment de tout souci de productivité.

Nous touchons là l'une des originalités des parcs français, leur lien avec l'aménagement du territoire et la mise en place de nouvelles formules de gestion des zones déshéritées et en voie d'abandon. Une nouvelle fonction de l'espace rural (détente, loisirs des urbains,...) semble alors être attribuée aux terres agricoles vues comme marginales.

L'histoire de ce Parc, objet de polémiques qui, tour à tour, a été qualifié de " parodie de parc national ", de "parc pas comme les autres » et auquel on ne prédisait qu'une courte existence, montre combien cette institution n'est, en fait, qu'une sorte de résurrection d'idées depuis longtemps oubliées : dans la seconde moitié du XIX ${ }^{\mathrm{e}}$ siècle et au début du $\mathrm{xx}^{\mathrm{e}}$ siècle, une partie minoritaire du Corps forestier mettait à mal le triomphalisme écologique au profit d'une conception sociale de la protection. Elle voulait, en s'opposant aux forestiers autoritaires qui n'hésitaient pas à exproprier les paysans pour reforester et protéger, faire "l'économie du protectionnisme biologique». Ces idées furent entérinées avec la création de ce qui fut en 1913 "le Parc de l'Oisans", territoire aujourd'hui inclus dans les limites du Parc national de la Vanoise.

Avec le Parc national des Cévennes, quelque soixante-dix ans plus tard, ce courant socio-économique de la protection émerge à nouveau.

2. Au terme de cette rétrospective qui souligne l'originalité et l'orthodoxie de l'institution, nous envisageons les stratégies et la cohérence interne du Parc et la façon dont il est perçu, voire utilisé, par les agriculteurs. L'analyse nous rappelle combien un Parc est un " produit social » à l'image de tout espace, un lieu de tensions et de contradictions. Les comptes-rendus des débats des Conseils d'administration suffisent à apprécier la vérité de ce propos.

Mais elle montre aussi que les tensions et les conflits n'opposent pas seulement société urbaine et société rurale, la première cherchant à déposséder la seconde en s'appuyant sur l'État. Les contradictions se situent également à l'intérieur des deux mondes. Nous nous proposons d'analyser celles caractérisant le monde rural cévenol.

Dès l'origine de l'histoire de ce Parc, il y a division entre ceux qui, en 1956, adhérent au projet - demande à l'unanimité de la création d'un Parc national par le Conseil général de la Lozère (il faut voir là l'influence de nouvelles élites locales, commerçants et entrepreneurs attentifs au phénomène touristique) et ceux (agriculteurs essentiellement) qui s'opposent au projet associant Parc national avec «faune sauvage » dont ils se font une représentation très particulière (bête du Gévaudan, chasse des nuisibles) et avec « réserve d'indiens » dans laquelle ils craignent d'être parqués. 

essoufflement. Le manque de crédit se pose avec acuité et rend la tâche de l'Établissement gestionnaire délicate, confronté à la multiplicité de ses objectifs. Les financements semblent de plus en plus s'orienter vers les activités d'accueil ; aussi les agriculteurs se sentent-ils lésés, comme trahis par une institution qui semblait avoir pris leur parti. Certains crient au «Parc folklore » et réactualisent les sentiments des années soixante-dix : «Le Parc, protecteur de la nature, est du côté des touristes plutôt que du côté des éleveurs ». D'autres reprochent à l'Établissement Public ses divers financements pour les réintroductions faunistiques, par exemple, et estiment qu'il ferait mieux de s'occuper d'eux. 
36 Il est vrai que, en lançant ce "pari agricole", le Parc a développé ses propres contradictions. Le postulat de l'agriculteur meilleur gestionnaire des ressources naturelles n'est pas remis en cause, mais induit alors de gros efforts financiers, pour maintenir l'intégrité des sites malgré la construction de bâtiments agricoles modernes, pour compenser les dégâts engendrés par une faune sauvage réintroduite.

37 En définitive, l'environnement n'est perçu que comme une contrainte financière et n'est appréhendé qu'au travers du problème de la qualité des sites. La question de fond, de compatibilité entre systèmes de production et protection du milieu naturel (sol, flore, petite faune), est en grande partie occultée.

38 3. Le troisième temps de l'analyse concerne le Parc en tant que structure territoriale, sa cohérence externe, son vécu face aux sociétés locales.

39 La région Cévennes a toujours souffert de son écartèlement administratif entre le Gard, la Lozère, l'Ardèche et l'Aveyron. Le Parc a-t-il contribué à donner aux Cévennes la dimension régionale qui leur manquait? On espérait faire du Parc une expérience de micro-régionalisation. En réalité, réaliser la concertation entre les différentes politiques rurales auxquelles était soumis l'espace cévenol, et entre tous les partenaires concernés, n'était pas chose aisée :

40 - le Parc s'est trouvé face aux difficultés de gérer un espace contenu dans des limites très complexes, à la fois défavorisé dans son ensemble, ce qui a suscité un projet national particulier, et hétérogène, couvrant des territoires dynamiques (comme le Causse Méjean) et des territoires en marge. Son aide sélective en faveur des agriculteurs dynamiques n'a pas compensé la division spatiale, mais a contribué à l'affirmation des territoires. Par ailleurs, le pouvoir influent des personnalités lozériennes, tant sur la scène locale que nationale, voire européenne, a contribué à façonner davantage le parc de la Lozère que le Parc des Cévennes.

41 - malgré sa mise sous tutelle, le Parc reste "le territoire des autres ", c'est-à-dire celui des propriétaires privés, celui des communes, de l'office national des forêts... Cette faiblesse propre à tous les parcs nationaux français est accrue en Cévennes où la proportion des propriétaires privés est la plus importante et où près de la moitié d'entre eux sont absentéistes ;

42 - le territoire protégé, enfin, est soumis à de multiples logiques institutionnelles (superposition des différents corps décisionnels). La D.D.A., par exemple, continue ses actions indépendamment du Parc qui n'a alors d'emprise qu'en matière esthétique. Le manque d'articulation entre les différents partenaires en présence renforce la structuration territoriale plus qu'elle ne l'estompe et affaiblit la position régionale des Cévennes.

43 L'intégration du Parc à un système de gestion de l'espace micro-régional n'est pas encore gagnée. Elle est d'autant plus délicate que le Parc a toujours été pressenti comme une menace par diverses personnalités craignant pour leur fonction, que ses attributions réglementaires générales l'élèvent au rang de tuteur de tous les partenaires et qu'enfin, son objectif de protection est perçu comme une contrainte supplémentaire à un développement local difficile.

Un tel travail de recherche permet aisément de mesurer le poids des conditions externes sur lesquelles une structure comme le Parc, cet organe « venu d'ailleurs ", n'a guère d'emprise et qui, pourtant, constitue le cadre dans lequel toute initiative locale doit se déployer... 

champ de réflexion toujours plus convoité dans le cadre multidisciplinaire de recherches sur l'environnement. Premier Parc à introduire une vision élargie de la conservation de la nature - c'est-à-dire celle qui recouvre la préservation, l'entretien, l'utilisation durable, la restauration et l'amélioration du milieu naturel -, il est l'un des territoires où apparaît avec évidence la nécessaire prise en compte des facteurs humains dans toute étude écologique et la nécessaire inclusion de l'analyse des ressources et du milieu de vie dans la réflexion sur les sociétés.

L'ancrage du P.I.R.E.N. qui associe sciences humaines et sciences de la nature sur cet espace en est la meilleure illustration. Reposant sur le principe du " mieux connaître pour mieux gérer ", ce programme se met au service des communautés locales et nationales désireuses d'innover sur le plan social et économique, en intégrant les connaissances écologiques aux décisions d'aménagement du territoire, aidant ainsi à concilier développement et protection de la nature.

De telles recherches doivent être poursuivies en faveur d'une "gestion écologique ", c'est-à-dire une gestion qui «favorise le maintien d'une diversité des communautés d'êtres vivants et garantirait une productivité biologique globale élevée au meilleur coût énergétique, tout en satisfaisant au moindre coût économique les besoins sociaux, culturels, et esthétiques diversifiés de la société $»^{4}$.

\section{NOTES}

1. Position de thèse. MOUSSET Sylvie, 1990. Nature paysannée, nature paysagère. De la protection à la gestion agricole d'un espace rural de moyenne montagne : l'exemple du Parc national des Cévennes. Thèse de $3^{\mathrm{e}}$ cycle, Université de Paris I, 227 pages + annexes.

2. Le plus récent date de 1990 : la profession agricole est accusée de détruire l'environnement - au sens qualité du milieu de vie (eau en particulier). Le principe du pollueur/payeur est relancé.

3. LEYNAUD Émile, 1982. «Les Parcs nationaux, territoires des autres », Espace géographique, 2, Paris, pp. 127- 138.

4. LEFEUVRE Jean-Claude, 1990. Les territoires du patrimoine, Dossiers des Séminaires

T.T.S., Ministère de l'Équipement.

\section{RÉSUMÉS}

Problématique et points forts d'un travail de recherche sur le Parc national des Cévennes, seul parc national habité qui fut amené à prendre des initiatives dans le domaine agricole. Quel est le 
rôle institutionnel du Parc dans l'évolution des hautes terres cévenoles? Comment est abordé le difficile compromis entre une mission de protection de la nature d'une part, et une mission de développement agricole d'autre part?

Protection of the countryside and agriculture in the Cevennes National Park Main findings of a research work on the Cevennes National Park concerning the difficult balance between the protection of the countryside and a concerted agricultural development.

INDEX

Mots-clés : Protection de la nature, parc national, Gestion écologique

Keywords : Cévennes, National Park, Cevennes, Protection of the countryside, Ecological management

\section{AUTEUR}

\section{SYLVIE MOUSSET}

Docteur de $3^{e}$ cycle en géographie, elle mène des recherches sur la protection de la nature et la gestion agricole dans le Parc national des Cévennes. 